\title{
Erratum \\ Endothelin-1 in plasma, cisternal CSF and microdialysate following aneurysmal SAH
}

\author{
S. Kästner, M. F. Oertel, W. Scharbrodt, M. Krause, D.-K. Böker, and W. Deinsberger [Acta Neurochir (Wien) \\ (2005) 147: 1271-1279].
}

DOI 10.1007/s00701-005-0633-0

On pape 1279 the wrong comment was printed. Below the correct version.

\section{Comment}

Kästner et al. determined the ET-1 level in the plasma, cerebrospinal fluid (CSF) and interstitial space of the brain parenchyma by microdialysis in patients after subarachnoid hemorrhage (SAH). From 20 patients after SAH included in the study (with heterogeneous Fisher and WFNS grade) 7 developed cerebral vasospasm (CVS), which was defined by a mean flow velocity of the middle cerebral artery of more than $120 \mathrm{~cm} / \mathrm{sec}$ or clinical deterioration.

The authors reproduced the well known findings that the plasma level of ET-1 does not correlate with an SAH or the development of CVS and that the CSF levels of ET-1 are increasing in most patients after SAH, which is markedly pronounced in patients developing CVS.

The innovative aspect in the present manuscript is the determination of ET-1 in the brain parenchyma by microdialysis after SAH. This level was significantly higher than the CSF level but showed no significant changes during the observation period even in cases with cerebral infraction. However, an antidromic (although not statistically significant) time course of the ET-1 concentrations was observed in patients with and without CVS on the days 3 and 4 after
SAH (Fig. 3), which was unfortunately not further highlighted and discussed by the authors.

ET-1 represents (at least since the results of the clinical study with clazosentan are available (Vajkoczy et al. [1])) a key factor in the pathophysiological sequence after SAH, leading to CVS. Investigations concerning potential sources of ET-1 after SAH in the cerebrospinal space (e.g. brain parenchyma) are, therefore, of considerable scientific value and may demonstrate new treatment or prophylaxis strategies for CVS. The present data are, therefore, in spite of their preliminary character of scientific interest. Additionally, this study may encourage further investigations to characterize the time course of the ET-metabolites in the brain parenchyma during the development of CVS after SAH in a well defined collective with a sufficient amount of patients.

\section{Reference}

1. Vajkoczy P, Meyer B, Weidauer S, Raabe A, Thome C, Ringel F, Breu V, Schmiedek P (2005) Clazosentan (AXV-034343), a selective endothelin A receptor antagonist, in the prevention of cerebral vasospasm following severe aneurysmal subarachnoid hemorrhage: results of a randomized, double-blind, placebo-controlled, multicenter phase IIa study. J Neurosurg 103(1): 9-17

V. Seifert and H. Vatter

Frankfurt 